\title{
Giant Choledochal Cyst in an Adult at a Teaching Hospital in South-Western Uganda: A Case Report
}

\author{
Mvuyo Maqhawe Sikhondze (iD) \\ Carlos Cabrera Dreque' \\ Edson Tayebwa' \\ Gotharido Tumubugane' \\ Charles Newton Odongo' \\ Eugene Ogwang ${ }^{2}$ \\ 'Department of Surgery, Mbarara \\ University of Science and Technology, \\ Mbarara City, South-Western Uganda, \\ Uganda; ${ }^{2}$ Department of Radiology, \\ Mbarara University of Science and \\ Technology, Mbarara City, South- \\ Western Uganda, Uganda
}

Correspondence: Mvuyo Maqhawe Sikhondze

Department of Surgery, Mbarara University of Science and Technology,

Mbarara City, South-Western Uganda, Uganda

Tel +256485420785

Fax +256485420782

Email maqhawesikhondze@yahoo.co.uk
Background: Choledochal cysts are rare congenital malformations characterized by cystic dilatations of the biliary tree. They are more prevalent in East Asian populations, but uncommon in western countries, and scarcely reported in African nations. They are classically diagnosed in children, and only $20-30 \%$ of cases are diagnosed in adulthood. Giant choledochal cysts are those with sizes exceeding $10 \mathrm{~cm}$, and are extremely rare with a few reported in the literature, thus the need for this case report.

Case: A 25-year-old Ugandan woman presented with an 8-month history of abdominal pain, jaundice and progressively increasing right upper quadrant abdominal mass. She underwent trans-abdominal ultrasonography that revealed a large, well-defined, echo-free mass in the right upper quadrant, extending to the lumbar region. Abdominal computed tomography (CT) revealed a huge thin-walled, cystic mass rising from the right wall of common hepatic duct, displacing the surrounding structures and no visible common bile duct. Complete cyst excision of a giant choledochal cyst type IVa was performed without decompression, in addition to cholecystectomy and reconstruction with hepaticojejunostomy and jejunojejunostomy anastomoses.

Conclusion: Giant choledochal cysts are a very rare pathology worldwide. Diagnosis can still be made using ultrasound and CT where more advanced imaging modalities like endoscopic retrograde cholangiopancreatography (ERCP) are not available. Complete surgical resection of giant choledochal cysts is surgically challenging, but essential to relieve symptoms and prevent malignant transformation. To the best of our knowledge, this is the first reported case in the literature, of a giant choledochal cyst that was successfully treated in an African nation.

Keywords: giant choledochal cyst, choledochal cyst, Todani's classification

\section{Introduction}

Choledochal cysts are uncommon congenital malformations of the biliary system characterized by a varying degree of cystic dilatations of the intrahepatic biliary ducts, extrahepatic biliary tree, or both. ${ }^{1}$ Giant choledochal cysts are those with sizes exceeding $10 \mathrm{~cm}$, and are extremely rare, with a few reported in the literature (Table 1). ${ }^{2}$ The precise cause of choledochal cysts is not known, but approximately $30 \%$ to $70 \%$ are linked to the presence of an anomalous pancreaticobiliary junction (APBJ), making it the most widely accepted aetiological theory. ${ }^{3}$ The incidence of choledochal cysts is highest in Asian nations, and is reported to be as high as 1:1000 in the Japanese population. In Western nations, the incidence ranges from $1: 100,000$ to $1: 150,000$ in the United States, to 1 in 2 million in the United Kingdom, but there is a paucity of data on choledochal cysts in African nations. ${ }^{4}$ 
Table I Giant Choledochal Cyst Publications in the Literature

\begin{tabular}{|l|c|l|l|}
\hline Author, Year Published & Cyst Type & Size in Centimeters $\mathbf{( c m})$ & Country \\
\hline Kumar et al, $2021^{16}$ & I and IV & Median cyst diameter: I5 cm (range, I0-20 cm) & India \\
Harikrishnan et al, $2020^{11}$ & IVA & $23 \mathrm{~cm} \times 15 \mathrm{~cm}$ & India \\
Yurttutan et al, $2016^{12}$ & I & $16 \mathrm{~cm}$ & Turkey \\
Khare et al, $2015^{15}$ & I & $15 \mathrm{~cm} \times 18 \mathrm{~cm} \times 9 \mathrm{~cm}$ & India \\
Anand et al, $2013^{2}$ & I and IV & Mean: $14.2 \mathrm{~cm}$; Range: $12-20 \mathrm{~cm}$ & India \\
Holland and Childs, $1996^{13}$ & I & $30 \mathrm{~cm}$ & Australia \\
Farrell $1959^{14}$ & I & Volume: $2600 \mathrm{cubic} \mathrm{cm}$ & USA. \\
\hline
\end{tabular}

They are classically diagnosed in children, and only $20-30 \%$ of cases diagnosed in adulthood, with female predominance. They are most frequently categorized into five main types based on anatomical configuration. Type I is cystic or fusiform dilatation limited to the extrahepatic bile ducts. Type II describes extrahepatic bile duct saccular diverticulum, while type III is choledochocele. Type IVa is presence of intrahepatic and extrahepatic cysts, type IVb is multiple dilatations in the extrahepatic bile ducts only, and type V is Caroli's disease, involving multiple dilatations of intrahepatic bile ducts only. Type I and IV are the most common in children and adults, respectively. ${ }^{5-7}$ Surgical excision and bilio-enteric anastomosis are the mainstay treatment modality for choledochal cysts to prevent malignant transformation. ${ }^{8,9}$ We report a case of a 25 -year-old woman who was diagnosed and underwent successful surgical management for a type IVa giant choledochal cyst, in a resource limited setting of southwestern Uganda.

\section{Case Presentation}

\section{Patient Information}

A 25-year-old female patient who presented with an 8month history of dull, non-radiating abdominal pain, and a progressively increasing mass in the right upper quadrant. She also noticed yellow discoloration of the eyes and body itching. She reported history of passing dark urine, but had not noticed clay-coloured stool. She denied any episodes of fever or chills, nausea and vomiting or recent weight loss. There was no significant medical history. She was from a rural part of Uganda and started soliciting medical attention two months after the symptoms initiated, and was driven mainly by the development of abdominal pain and the mass. At the time of presentation to our institution, she had been referred to two other hospitals, over a five-month period. Ours was the third, and nearly $100 \mathrm{~km}$ away from her home. The other facilities she had visited had no further diagnostic equipment available, other than baseline blood work and ultrasonography that were inconclusive.

\section{Clinical Findings}

On physical examination, she had obvious jaundice with a large mass arising from the right hypochondriac region, occupying the right side of her abdomen and slightly crossing the midline. The mass was soft, non-mobile, with a smooth surface and slightly tender on palpation, measuring approximately $20 \times 20 \mathrm{~cm}$, and gave the impression to be separate from the liver. There were no collateral vessels, no periumbilical mass, and no palpable cervical lymphnodes (Figure 1).

\section{Diagnostic Assessments}

Blood work included complete blood count, serum electrolytes, liver function tests, INR and hepatitis, and tumour markers (Table 2). Ultrasound showed a large, well-defined, echofree mass in the right hypochondriac region, extending to the lumbar region, indenting with the liver laterally. A computed tomography (CT) scan was requested shortly after admission and it revealed a huge thin-walled, non-enhancing cystic mass rising from the right wall of common hepatic duct, displacing the surrounding structures including right kidney, IVC, portal vein, pancreas, mesentery and bowel loops, outwards. Intrahepatic ducts are dilated and common bile duct (CBD) not visualized (Figure 2).

\section{Therapeutic Intervention}

She underwent an exploratory laparotomy via an extended midline incision. Upon entry to the peritoneum, there were no ascites and no lymph nodes. Greater omentum was adherent and covering the cyst anteriorly, the duodenum adhered and outstretched over the left anterior-medial aspect of the cyst. The pancreas was thinned-out and adhered to the medial wall of the cyst. After prolonged and careful latero-medial dissection, these attachments were separated from the mass, without 


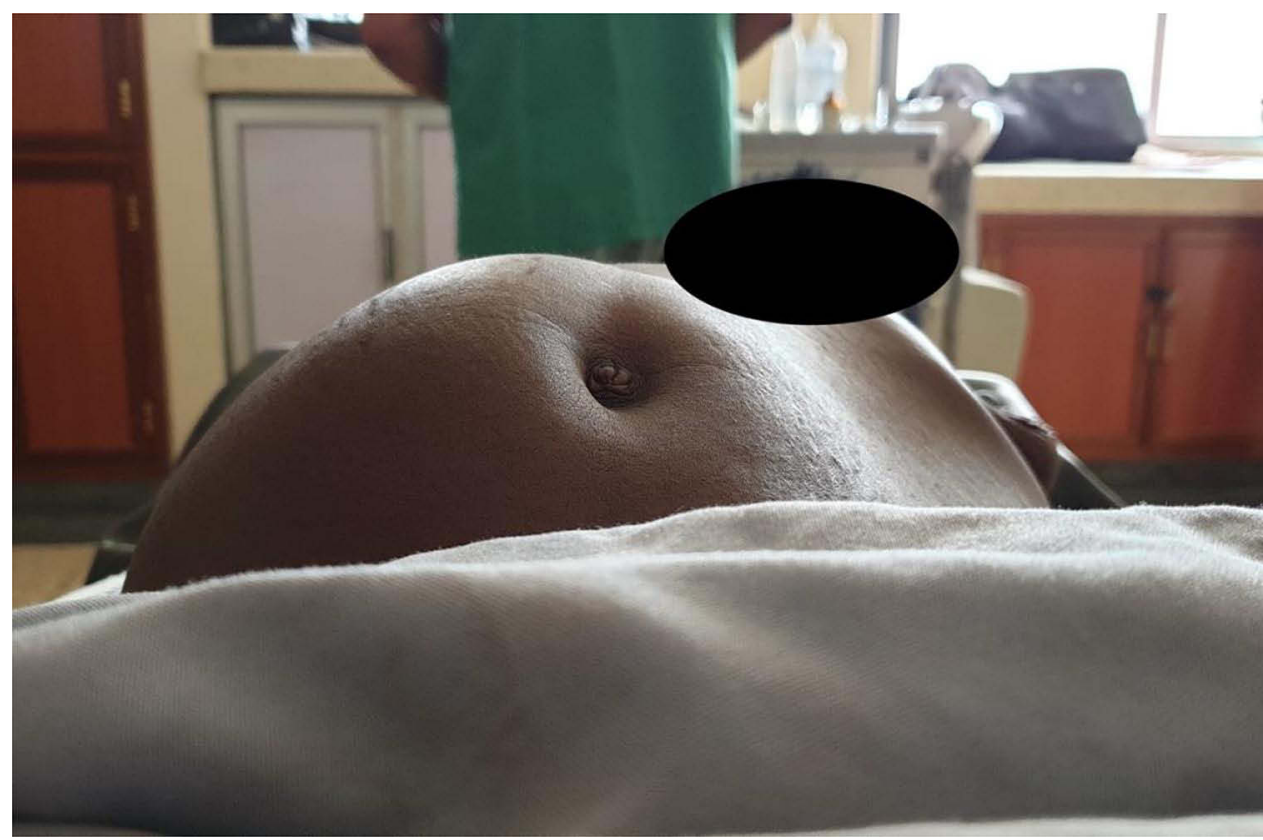

Figure I Pre-operative view of abdomen.

discernible injury, using blunt dissection and monopolar cautery. This allowed clearer visualization of the mass, noted to be a very large diffuse extrahepatic biliary cyst measuring
$20 \times 15 \times 20 \mathrm{~cm}$, mainly involving the common bile duct (CBD). The cyst had a blind-ending distal border, with no obvious tailing or tapering. Dissection was then carried out

Table 2 Laboratory Tests

\begin{tabular}{|c|c|c|c|}
\hline Parameter & $\begin{array}{l}\text { Pre-Operative Laboratory } \\
\text { Values }\end{array}$ & $\begin{array}{l}\text { Laboratory Values at Last } \\
\text { Follow-Up }\end{array}$ & $\begin{array}{l}\text { Reference Range of MRRH } \\
\text { Laboratory }\end{array}$ \\
\hline \multicolumn{4}{|l|}{ Blood } \\
\hline White blood count $\left(10^{9} / \mathrm{L}\right)$ & 7.08 & 5.13 & $4.00-10.00$ \\
\hline Haemoglobin (g/dL) & 11.5 & 12.0 & $11.0-15.0$ \\
\hline Platelets $\left(10^{9} / \mathrm{L}\right)$ & 519 & 513 & $100-300$ \\
\hline Bilirubin- total $(\mathrm{mg} / \mathrm{dL})$ & 9.0 & 0.7 & $0.1-1$ \\
\hline Bilirubin- direct (mg/dL) & 5.20 & 0.15 & $0-0.2$ \\
\hline Alanine Transaminase $(\mathrm{u} / \mathrm{l})$ & 96.9 & 21 & $0-37$ \\
\hline Aspartate Transaminase $(\mathrm{u} / \mathrm{l})$ & 38.8 & 15 & $0-42$ \\
\hline Alkaline Phosphatase (u/l) & 973.5 & 220 & $60-306$ \\
\hline Prothrombin time $(\mathrm{sec})$ & 15.3 & Not repeated & $11-15$ \\
\hline International Normalized Ratio & 1.24 & Not repeated & $0.8-1.2$ Up to 3 (on Warfarin) \\
\hline$(\mathrm{INR})^{*}$ & & & \\
\hline CA 19-9* & 10 & & $0-36$ \\
\hline CEA $(\mathrm{ng} / \mathrm{mL})^{*}$ & I.I & & $<2.5$ \\
\hline Hepatitis B & Negative & Not repeated & \\
\hline \multicolumn{4}{|l|}{ Urinalysis } \\
\hline Proteins & Proteinuria ++ & & \\
\hline Leukocytes & + & & \\
\hline Bilirubin & ++ & & \\
\hline
\end{tabular}

Note: *Investigations not available at MRRH, done in a private laboratory.

Abbreviation: MRRH, Mbarara Regional Referral Hospital. 

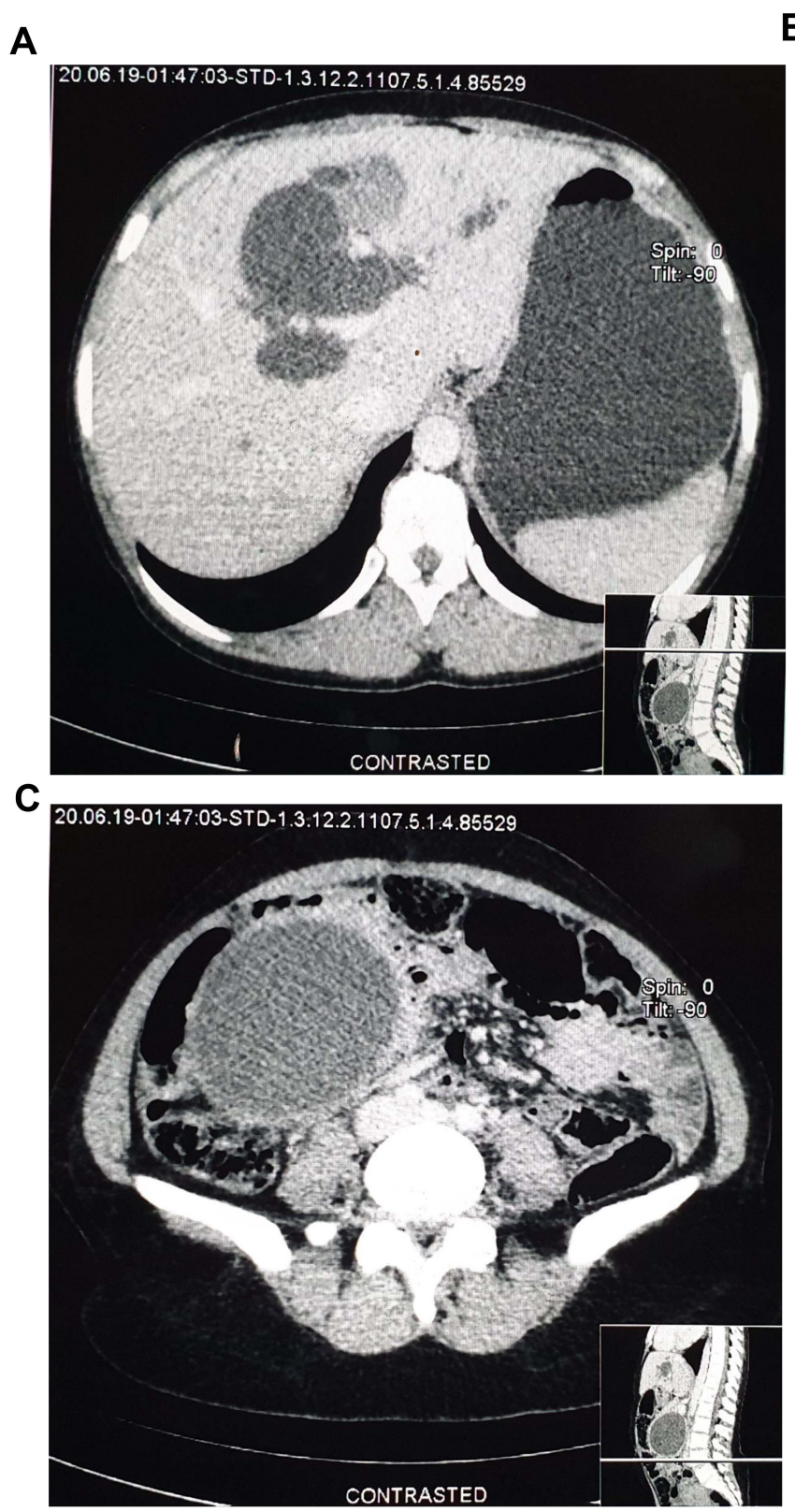

B

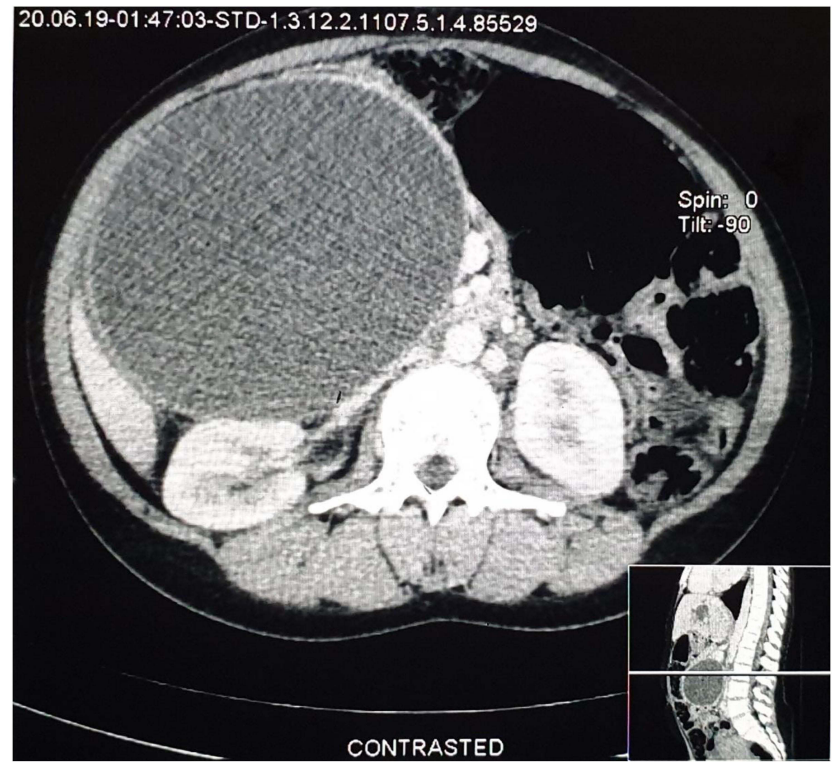

Figure 2 CT scan showing the huge thin-walled, non-enhancing cystic mass rising from the right wall of common hepatic duct. (A) Shows intrahepatic duct dilatation. (B) Shows the cyst at the widest antero-posterior diameter. (C) Shows the distal end of the cyst.

proximally towards the hepatic ducts. With the gall bladder notably dilated and cystic duct (CD) forming the superiorlateral border of the cyst, cholecystectomy was also done (Figures 3 and 4). Both hepatic ducts and the common hepatic duct (CHD) were also dilated. The cyst was transected $1 \mathrm{~cm}$ proximal to the confluence of the $\mathrm{CHD}$ and the $\mathrm{CD}$, and complete extrahepatic cyst excision was performed without prior decompression of the mass. Biliary reconstruction was achieved through hepaticojejunostomy and jejunojejunostomy anastomosis. A drain was left in proximity to the hepaticojejunostomy anastomosis and removed 7 days later, after having spent two days without any collection. The specimen was sent for histopathology examination and it was reported to contain chronic inflammatory infiltrate with no evidence of malignancy. The patient had an uneventful post-operative period and was discharged on the tenth day after surgery.

\section{Follow-Up and Outcomes}

Patient was reviewed 2 weeks after discharge and reported improvement, with no new complaints. Her control liver function tests showed reduction in liver enzymes. At the last review, the patient was 8 months post-operative, with 

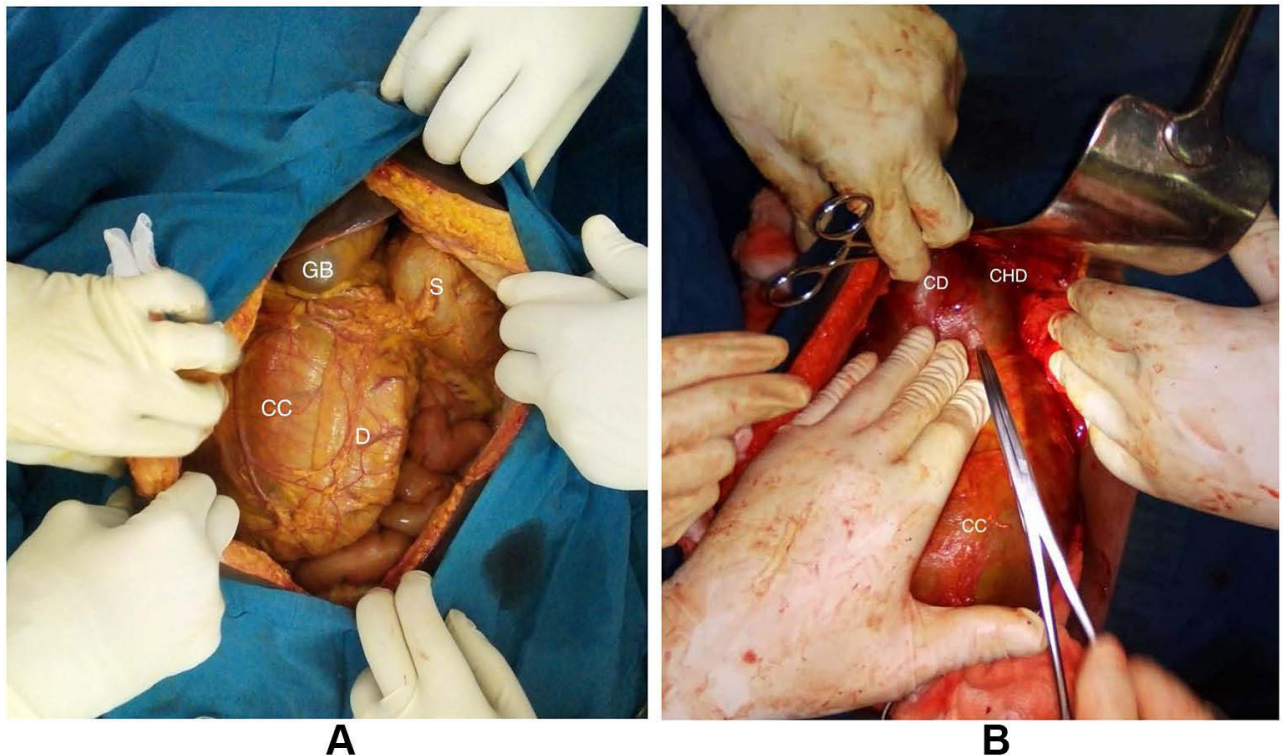

Figure 3 Intraoperative views. (A) Shows the biliary cyst with the duodenum outstretched over it. (B) Shows the cyst with cystic duct and common hepatic duct dilatation. Abbreviations: GB, gallbladder; CC, choledochal cyst; D, duodenum; S, stomach; CD, cystic duct; CHD, common hepatic duct.

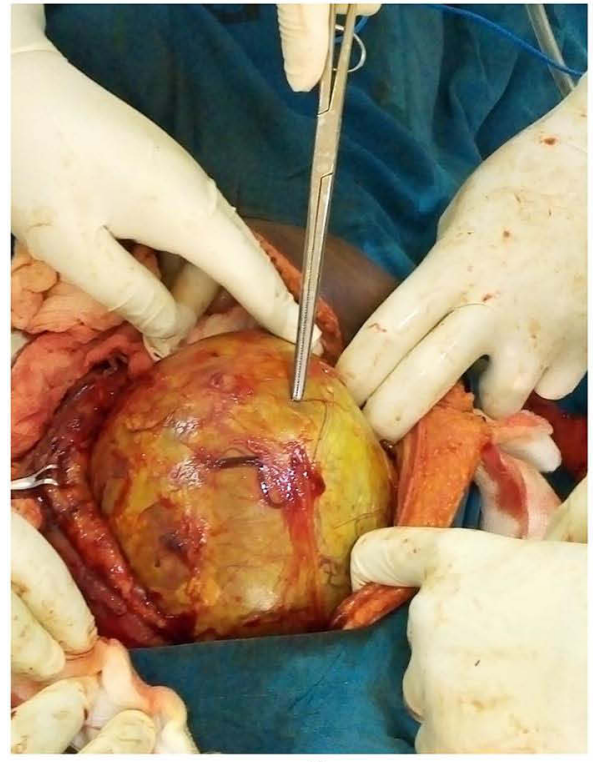

A

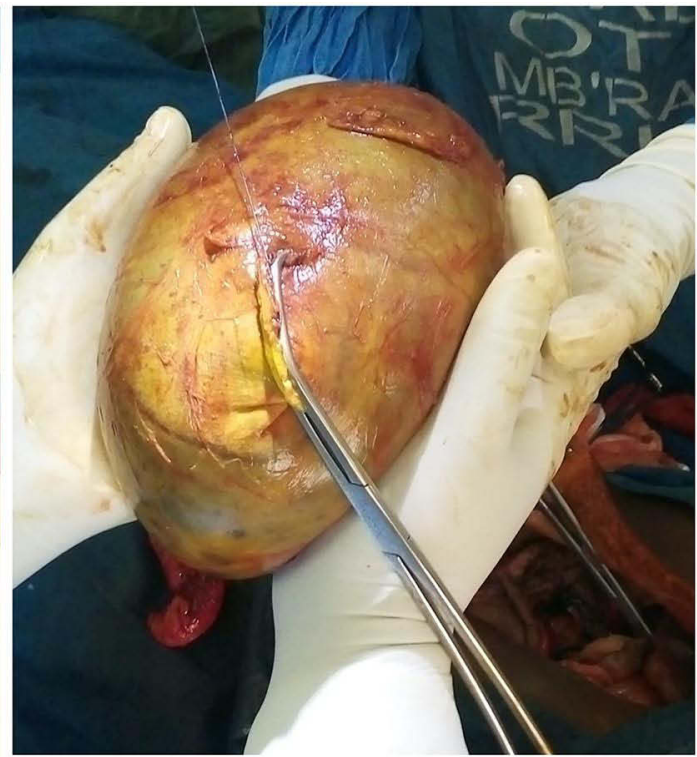

B

Figure 4 Intraoperative pictures of the huge choledochal cyst. (A) Cyst after being dissected and free of adjacent structures. (B) Intraoperative picture of the huge choledochal cyst being lifted after excision.

no complications and the jaundice had cleared. She was doing well and had experienced resolution of the abdominal pain and jaundice.

\section{Discussion}

Choledochal cysts are rare congenital malformations depicted as cystic dilatations of the intra- and/or extrahepatic biliary system. ${ }^{1}$ Choledochal cysts are more prevalent in East Asian populations, but uncommon in western countries, and scarcely reported in African nations. ${ }^{4}$ Our patient is an African woman from southwestern Uganda, living approximately $100 \mathrm{~km}$ from our health facility.

The Todani classification is the most widely used and categorizes choledochal cysts into 5 subtypes based on their anatomical configurations. Type I is cystic or 
fusiform dilatation limited to the extrahepatic bile ducts, and is further subdivided into type Ia - cystic dilatation of the CBD, type Ib - segmental/focal, and type Ic - fusiform. Type II describes extrahepatic bile duct saccular diverticulum, type III is choledochocele. Type IVa is presence of intrahepatic and extrahepatic cysts, type IVb is multiple dilatations in the extrahepatic bile ducts only, and type $\mathrm{V}$ is Caroli's disease, involving multiple dilatations of intrahepatic bile ducts only. Type I and IV occur more the most common in children and adults, representing $50 \%$ to $80 \%$ and $15 \%$ to $50 \%$, respectively. ${ }^{6,7}$ In the present case, the patient had a type IVa choledochal cyst characterized by dilatation of both the extrahepatic and intrahepatic bile ducts, with associated cystic dilatation of the CBD, cystic duct, common hepatic ducts and both hepatic ducts.

The most widely accepted theory regarding the aetiology of choledochal cysts is the presence of an anomalous pancreaticobiliary junction (APBJ). ${ }^{3}$ The APBJ is the point 1 to $2 \mathrm{~cm}$ proximal to the sphincter of Oddi where the biliary and pancreatic duct join, and the anomaly allows backflow and mixing of biliary and pancreatic secretions. Pancreatic enzymes are activated and an increase in pressures results in dilatation, inflammation, epithelial damage, dysplasia and can subsequently cause malignancy of the biliary tree. 8

The patterns of presentation vary between adults and children, with adults more likely to present with biliary or pancreatic symptoms and abdominal pain, and obstructive jaundice being the herald symptom in children. Adult patients frequently present with unspecific symptomatology including ranging from right upper quadrant abdominal pain, jaundice, nausea, vomiting to fever. ${ }^{10}$ The patient described in this report presented with right upper quadrant abdominal pain, jaundice and an abdominal mass, which are considered uncommon, constituting the classic triad of presentation for choledochal cysts seen in only $20 \%$ of cases of choledochal cysts. ${ }^{5,7}$ This patient's mass was clearly visible on inspection and also causing significant abdominal heaviness and asymmetry.

Imaging techniques like magnetic resonance cholangiopancreatography (MRCP), endoscopic retrograde cholangiopancreatography (ERCP) and endoscopic ultrasound superseded other imaging techniques in the diagnosis of choledochal cysts, and are essential to preoperatively define biliary anatomy. ${ }^{7}$ Nonetheless, these imaging modalities are not available in limited resource settings like ours, which is why she presented with multiple ultrasound scans from different facilities, all in an attempt to get to a final diagnosis. She finally had a computed tomography (CT) scan done at our centre, six months after she started seeking medical attention, while the abdominal mass, jaundice and pain progressively increased.

There are variations in biliary cyst sizes, and those with are greater than $10 \mathrm{~cm}$ are referred to as giant choledochal cysts. ${ }^{2}$ There are few reported cases of giant choledochal cysts currently found in the literature, due to the very rare nature of the condition (Table 1). ${ }^{2,11-16}$ Our patient had gross dilatation of the extrahepatic biliary tree found to be measuring $20 \times 15 \times 20$, thus fitting the criteria of a giant choledochal cyst.

Surgical excision remains the mainstay treatment modality recommended for choledochal cysts and has been reported to be protective against malignant transformation. ${ }^{9,17}$ Type I and IV are managed with complete excision of the choledochus with cholecystectomy, and restoration of bile flow via a bilioenteric anastomosis. Simple excision is done for type II cysts, and sphincterotomy for type III cystic lesion. Caroli's disease may require partial hepatic resection. ${ }^{7}$ Giant choledochal cysts have been reported to be surgically challenging, with risks of postoperative complications like biliary leakage, pancreatitis, pancreatic leakage and fistula. ${ }^{11,16}$ The occurrence of pancreatic complications would have possibly required high dependency care (HDU) or even intensive care unit admission depending on the severity, which could have been a challenge since our institution has frequent unavailability of ICU space because of a limited 8-bed capacity ICU to cover the whole south-western region of the country, serving a population of over 8 million. The operation on our patient included careful dissection of all adherent structures including the duodenum and pancreas, and complete extrahepatic cyst excision, cholecystectomy and biliary reconstruction achieved through hepaticojejunostomy and jejunojejunostomy anastomoses. The histology results were received two weeks after surgery, which is not unusual in our setting due to histopathology backlogs and scarcity of pathologists. After the review at 8 months post-op, she was improving with no new complaints and adviced on yearly review as she is still at risk of malignancy, even after excision. ${ }^{7}$

\section{Conclusion}

Choledochal cysts are uncommon congenital abnormality in adults, and thus need to be considered as a possible diagnosis in patients with jaundice. Giant choledochal cysts are an even more rare pathology worldwide. Diagnosis can still be made using ultrasound and CT in limited resource settings, where more modern imaging modalities like ERCP are not available. 
Complete surgical resection of giant choledochal cysts is surgically challenging, but essential to relieve symptoms and prevent malignant transformation. To the best of our knowledge, this is the first reported case in the literature of a giant choledochal cyst that was successfully treated in an African nation.

\section{Ethical Approval}

Ethical approval was obtained from Mbarara University of Science and Technology Research Ethics Committee (REC).

\section{Consent}

Written informed consent was obtained from the patient for publication of this case report and accompanying images and is available for review by the Editor-in-Chief of this journal on request.

\section{Acknowledgments}

We acknowledge the nurses, theatre staff, and other care providers of the surgical department of Mbarara Regional Referral Hospital, Mbarara, who participated in the care of the patient whose case is described in this report. Published with written consent of the patient.

\section{Author Contributions}

All authors made a significant contribution to the work reported, whether that is in the conception, study design, execution, acquisition of data, analysis and interpretation, or in all these areas; took part in drafting, revising or critically reviewing the article; gave final approval of the version to be published; have agreed on the journal to which the article has been submitted; and agree to be accountable for all aspects of the work.

\section{Disclosure}

The authors report no conflicts of interests in this work.

\section{References}

1. Al-Salem A. Choledochal cyst. In: An Illustrated Guide to Pediatric Surgery. Cham: Springer; 2014.

2. Anand U, Priyadarshi RN, Kumar B, et al. Diagnosis and management of giant choledochal cysts: complexities compared to smaller cysts. World J Gastroenterol. 2013;32(4):262-267. doi:10.1007/ s12664-013-0335-0

3. Babbitt D. [Congenital choledochal cysts: new etiological concept based on anomalous relationships of the common bile duct and pancreatic bulb]. Ann Radiol. 1969;12:231. French.

4. Baison G, Bonds MM, Helton WS, et al. Choledochal cysts: similarities and differences between Asian and Western countries. World $J$ Gastroenterol. 2019;25:3334-3343. doi:10.3748/wjg.v25.i26.3334

5. Soares KC, Kim Y, Spolverato G, et al. Presentation and clinical outcomes of choledochal cysts in children and adults: a multi-institutional analysis. JAMA Surg. 2015;150(6):577-584. doi:10.1001/ jamasurg.2015.0226

6. Todani T, Watanabe Y, Toki A, et al. Classification of congenital biliary cystic disease: special reference to type Ic and IVA cysts with primary ductal stricture. J Hepatobiliary Pancreat Surg. 2003;10 (5):340-344. doi:10.1007/s00534-002-0733-7

7. Hoilat GJ, John S. Choledochal Cyst. StatPearls; 2021.

8. Singham J, Yoshida EM, Scudamore CH. Choledochal cysts: part 1 of 3: classification and pathogenesis. Can J Surg. 2009;52(5):434440.

9. Ten Hove A, de Meijer VE, Hulscher JBF, et al. Meta-analysis of risk of developing malignancy in congenital choledochal malformation. Br J Surg. 2018;105(5):482-490. doi:10.1002/bjs. 10798

10. Gadelhak N, Shehta A, Hamed H. Diagnosis and management of choledochal cyst: 20 years of single center experience. World $J$ Gastroenterol. 2014;20(22):7061. doi:10.3748/wjg.v20.i22.7061

11. Harikrishnan S, Chandramohan SM, Chandramohan A. Giant choledochal cyst type 4A: a surgical challenge. Pan Afr Med J. 2020;37. doi:10.11604/pamj.2020.37.95.24811

12. Yurttutan N, Karakus SC, Koku N, et al. A giant choledochal cyst in infancy: a case report. Korean $J$ Pediatr. 2016;59(5):239. doi:10.3345/kjp.2016.59.5.239

13. Holland A, Childs P. Case report: giant choledochal cyst. $J$ Gastroenterol Hepatol. 1996;11(9):847-849. doi:10.1111/j.14401746.1996.tb00091.x

14. Farrell WJ. Giant choledochal cyst: report of a case successfully treated by Roux-en-Y choledochocyst-jejunostomy. Ann Surg. 1959;150(2):313. doi:10.1097/00000658-195908000-00016

15. Khare MK, Dewangan M, Naik T, Mohanty MK. Giant choledochal cyst. Trop Gastroenterol. 2015;36(2):120-121. doi:10.7869/tg.265

16. Kumar S, Singla S, Kumar S, et al. Tactics, techniques, and challenges in the management of giant choledochal cyst in adolescents and adults: a retrospective cohort study. Langenbecks Arch Surg. 2021;406:1925-1933. doi:10.1007/s00423-021-02209-6

17. He X-D, Wang L, Liu W, et al. The risk of carcinogenesis in congenital choledochal cyst patients: an analysis of 214 cases. Ann Hepatol. 2014;13(6):819-826. doi:10.1016/S1665-2681(19)30985-8

International Medical Case Reports Journal

Dovepress

\section{Publish your work in this journal}

The International Medical Case Reports Journal is an international, peer-reviewed open-access journal publishing original case reports from all medical specialties. Previously unpublished medical posters are also accepted relating to any area of clinical or preclinical science. Submissions should not normally exceed 2,000 words or 4

published pages including figures, diagrams and references. The manuscript management system is completely online and includes a very quick and fair peer-review system, which is all easy to use. Visit http://www.dovepress.com/testimonials.php to read real quotes from published authors. 